\title{
Minimally Invasive Treatment With Platelet Rich Plasma In Patients With Knee Osteoarthritis
}

\author{
Tiberiu ${ }^{1}$, Copotoiu Sanda-Maria ${ }^{3}$ \\ 1 Department of Orthopedic Surgery and Traumatology I, County Emergency Clinical Hospital, Târgu-Mureș, România \\ 2 Department of Rheumatology and Physiotherapy, County Emergency Clinical Hospital, Târgu-Mureș, România \\ ${ }^{3}$ Department of ICU and Anesthesiology I, County Emergency Clinical Hospital, Târgu-Mureș, România
}

Albu Daniel-Emil1, Copotoiu Monica², Jeremias Cristina², Susca Mihaela², Melinte Răzvan¹, Bățagă

\begin{abstract}
Background: The main target of the structural damage in osteoarthritisis the hyaline cartilage. New options such as PRP (platelet rich plasma) may cause structural improvement of the cartilage. Objective: The aim of ourstudywas to evaluate the evolution of the patients with knee osteoarthritis on PRP treatment. Material and method: The patients with osteoarthritis were evaluated at enrolment and every three months by ultrasound knee ecography. A semi-quantitative knee ultrasound score was applied to monitor changes before and after the treatment. The score was composed by the presence of the following: bursitis (1 point), hypoechogenic changes at the level of the hyaline cartilage in the anterior transversal window (1 point), asymmetrical narrowing of the cartilage (1 point), the involvement of the medial cartilage in the longitudinal approach (1 point), the involvement of lateral cartilage in the longitudinal approach (1 point) and the presence of step up lesions - osteophytes ( 1 point The evaluation was performed independently by two separate physicians. Another ultrasound - trained doctor was analysing all the images. Results: Twenty - nine patients were included in our study; with one patient excluded due to masive bursitis. No differences were found concerning the pathological findings between the first two evaluators (p: 0.1250). So far, only nine patients have been evaluated at three months, and we were unable to find any improvements or worsening concerning the ultrasound alterations (p: 0.0625). The ultrasound lack of modifications was not correlated with the functional status of the subjects. Conclusions: In the short term, the local treatment showed no improvement on structural damage, but it improved the overall status of the patients (less pain, improved mobility, a better quality of life).
\end{abstract}

Keywords: Osteoarthritis PRP ecography knee cartilage

Received: 21 July 2015 / Accepted: 03 September 2015

\section{Introduction}

Osteoarthritis $(\mathrm{OA})$, characterized by a destruction of the articular hyaline cartilage, alterations to the subchondral bone and synovitis, is a common and debilitating condition found both among elderly patients and among the young. This pathology is mainly associated with pain and loss of joint mobility, two simptoms that undermines quality-of-life. The mechanisms responsible for the cartilage distruction and OA progression are very complex and at this point poorly understood. Articular cartilage has limited inherent healing capacity due to its avascular and alymphaticnature [1-5]. Currently available drugs, used for the treatement of $\mathrm{OA}$, such as nonsteroid and steroid anti-inflammatory drugs, analgesics chondroitin sulphate and hyaluronic acid are mainly directed toward the symptomatic relief (pain and inflammation) but they do little to none in reducing the cartilage destruction. Several surgical techniques are also available, including microfractures at the defect site, autologous grafts (mosaicplasty) and allograft osteochondraltransplantation [6-8]. However, the reported results with these procedures have been variable and are not guaranteed to prevent further progression of cartilage destruction $[1,2]$. Regarding this fact, current research is investigating new methods of promoting cartilage repair, one of which is based on the applications of growth

* Correspondence to: Daniel Emil Albu

E-mail: albu.daniel.e@gmail.com factors. The efficacy of growth factors in the treatment of cartilage destruction is related to the recruitment of chondrogenic cells, proliferation stimulation and cartilage matrix synthesis $[3,4]$. Platelet-rich plasma (PRP) is defined as an autologous concentration of human platelets in a small volume of plasma. These platelets are proven to be actively secreting protein growth factors that initiate mesenchymal tissue healing, cell proliferation and chondrogenesis. The abundance of growth factors contained in platelets led to be believed that PRP can augment or stimulate cartilage healing with the same biological healing process that normally occurs in the human body after different types of injuries $[3,4,9]$. The aim of our study was to follow the evolution of the patients with knee osteoarthritis on PRP treatement by doing periodical ecographic examinations and correlating these findings with patients clinical score.

\section{Material and method}

A prospective, interventional study was carried out in the Orthopedic Surgery and Traumatology Clinic I in collaboration with the Rheumatology and Physiotherapy Department, County Emergency Clinical Hospital, TârguMureș, România. Twentynine patients were included in our study (13 female and 16 male with a mean age of $50.34+/-15.50 y$ rsold); with one patient excluded due to massive bursitis; all of whom were diagnosed with stages I, II and III knee chondropathy (4 patients with grade I, 15 patients with grade II and 4 patients with grade III). 
The inclusion criteria were chronic knee pain in the last six to twelve months and radiological and ecographic signs of hyaline cartilage destruction while the exculsion criteria were thrombocytopenia, anemia $(\mathrm{Hb}, 10 \mathrm{~g} / \mathrm{dl})$, any history of active or past hematological disease, history of tumor or hematological malignant disease, active state of infection and active anticoagulant therapy and patients with stage IV chondropathy (indication for total knee replacement surgery). Body mass index was also taken into consideration( ranging $21.1-35.7 \mathrm{~kg} / \mathrm{m}^{2}$ ). All the patients included in this study did not follow any other treatment method other than PRP therapy and the use of anti-inflammatory drugs was not permitted from 7 days before the beginning of treatment to 7 days after the last treatment dose. This clinical trial was approved in accordance with the ethical standards of our hospital committee, and the informed consent was obtained from all off our patients before starting treatment.

The procedure for preparing PRP, described in this paper, was modified after several trials and according to the international literature $[4,5,11]$. The blood sample $(16-18$ $\mathrm{ml}$ of autologous venous blood) was drawn into two 9-ml vacutainer tubes (BD Vacutainer for plasma) each containing Sodium Heparin (17 UI/ml of blood). The obtained samples were gently agitated to thoroughly mix the blood with the anticoagulant and then centrifuged at $1500 \mathrm{rpm}$ for 5 minutes at room temperature (HettichZentrifugen EBA 20) resulting in three following layers: inferior layers composed of erythrocytes, the intermediate layer composed of leukocytes (the buffy coat) and the superior layer made up of plasma (PRP). The superior layers were then aspirated with two $5 \mathrm{ml}$ syringe, obtaining a final PRP product (5-6 $\mathrm{ml}$ ) used for the intra-articular injection within the first 30 minutes; regarding the fact that the platelets secrete $70 \%$ of their stored growth factors in the first 10-15 minutes and close to $100 \%$ in the first hour and can go on for about 8 days until they are depleted and die [10-12]. The injection was performed through a lateral approach of the knee joint, after disinfection of the skin, using sterile instruments and consumables. We repeated the procedure at one week interval for a total of three injections, all procedures being performed in the same office setting. Considering the various positive effects of PRP treatment in different clinical applications, we opted to use nonactivated PRP (it is shown that thrombin activated PRP inhibits chondrogenesis while nonactivatet PRP favorized bone and cartilage formation in vitro and in vivo) $[3,13]$.

The total time needed to prepare the platelet concentrate and performing the injection was approximately 7 minutes.

Before the treatment, all patients underwent base-line clinical evaluation, using the Tegner Lysholm Knee Scoring Scale (Scoring Scale: poor <65/ fair 65-83 / good 84-90 / excellent >90). The Tegner Lysholm Knee Scoring Scale evaliuates the existence of limp, the need of support, the locking sensation, the instability acquired, the pain re- sented by the patient, the swelling of the knee, the possibility of stair - climbing and squatting. The patients were investigated using non-weight-bearing $\mathrm{x}$-rays and ultrasound knee ecography. The outcome was then measured at four weeks and every three months afterwards.

Ultrasonography was used for the quantification of the inflammatory (joint effusion - bursitis) and structural (hyaline, cartilage thickness, osteophytes) lesions of the knee. Musculoskeletal ultrasound (US) was preferred to radiology - the presumed golden standard of knee osteoarthritis evaluation, because has the advantage of not being a 2-D examination, is dynamic, is not expansive, is reachable, is reproductable, has the capacity of showing additional soft tissue changes and has no contraindications, but it is dependent on the observer. That'swhy; two separate physicians performed the evaluation independently. Another ultrasound - trained senior doctor analyzed all the images. The senior doctor trained both physicians. Such biased results to be excluded [14-16,19].

The US examination was performed using the linear multi frequency probe (3 to $12 \mathrm{MHz}$ ) of the Phillips $\mathrm{HD}$ 7 US machine. All patients were examined with the same apparatus.

The US evaluation was performed according with the OMERACT recommendations and EULAR protocol of knee scanning and lesions definitions. The first target of US was to find cartilage changes so the patient's knee was completed flexed in order to reveal the window of knee hyaline cartilage. Axial and sagital images of medial and lateral cartilage were captured. In order to evaluate additional changes accompanying the OA's knee the patient was asked to flex the knee at an angle of $30^{\circ}$ and transversal and longitudinal images of knee were obtained [15-19].

A semi-quantitative knee ultrasound score was applied to monitor changes before and after the treatment. The score was composed by the presence of the following: bursitis (1 point) (Fig. 1), hypoechogenic changes at the level of the hyaline cartilage in the anterior transversal window (1 point) (Fig. 2), asymmetrical narrowing of the cartilage (1 point) (Fig. 3), the involvement of the medial cartilage in the longitudinal approach (1 point) (Fig. 4), the involvement of lateral cartilage in the longitudinal approach (1 point) and the presence of step up lesions - osteophytes (1 point) (Fig. 5). We summed the findings obtaining a maximum score of 6 with a minimum of 0 .

OMERACT lesion's definitions were used in order to assess the images. The joint effusion was scored if an anechoic or hypoechoic, compressible with no Doppler signal area was to be seen in the joint. Osteophytes were defined as "step up lesions" - cortical protrusions of the bone margin seen in two planes. The cartilage modifications were defined as gaining in the hypoechogenity and loosing in thickness [15,17-19].

The aim of the US score was not to be changed regarding the structural lesions and looking for a better outcome regarding the inflammatory ones (joint effusions). 


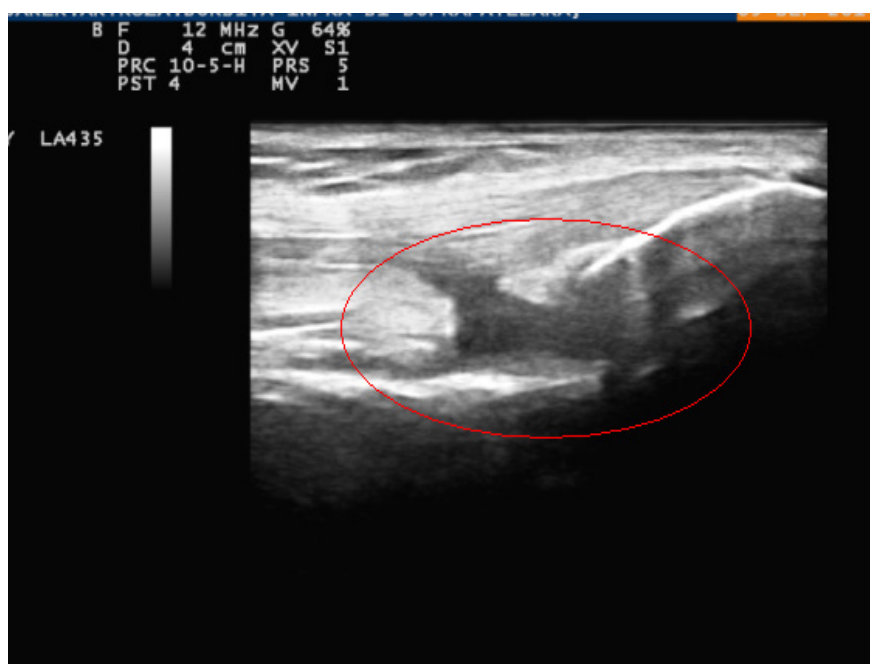

Fig. 1. Bursitis

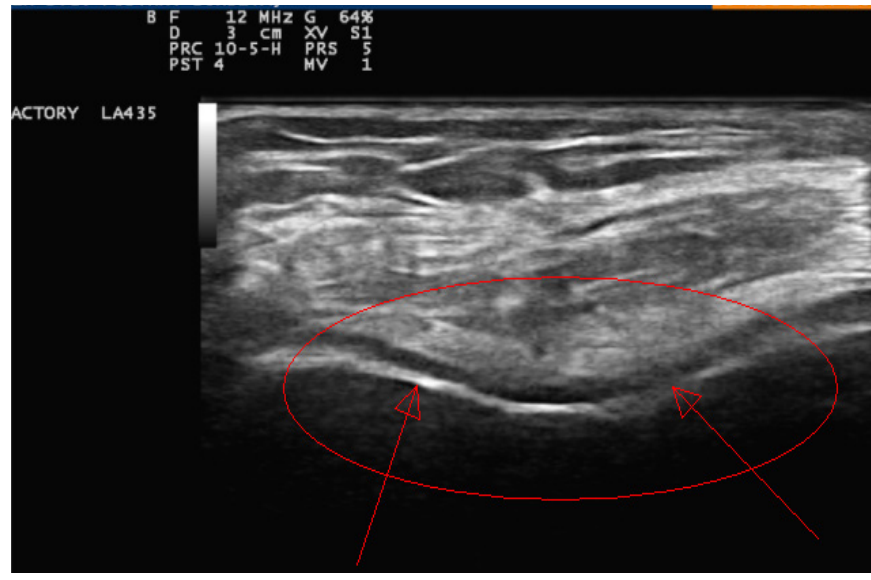

Fig. 3. Asymmetrical narrowing of the cartilage

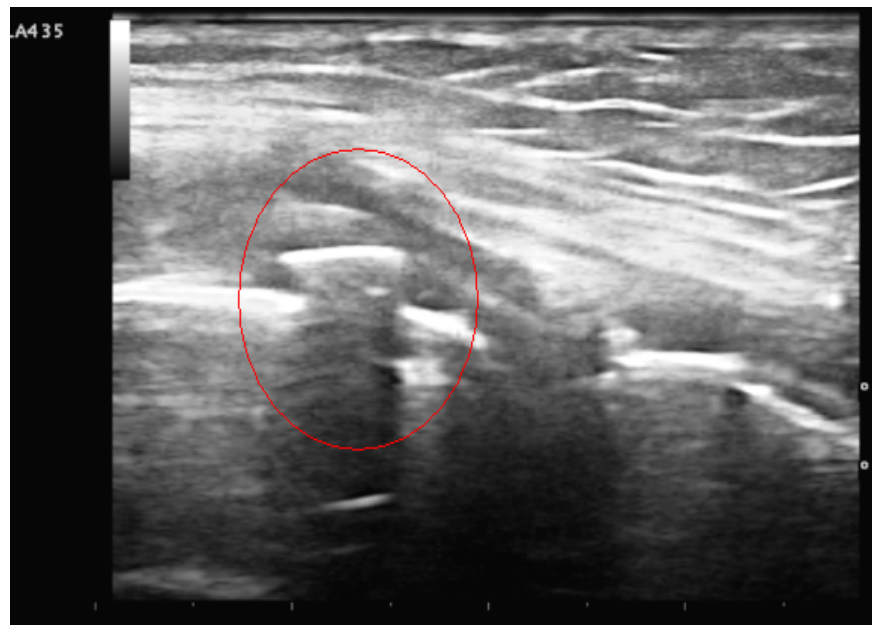

Fig. 5. Step up lesions

During the treatment no activities were prohibited and our patients did not participate in any physical therapy (to exclude any synergistic effect) and there was no specific post procedural exercise or any type of motion routine.

The data were analyzed using the Graph Pad Prism statistic software. The Wilcoxon matched pairs sign rang test, The Mann Whitney test, ANOVA test and Spearman correlation were applied to our data.

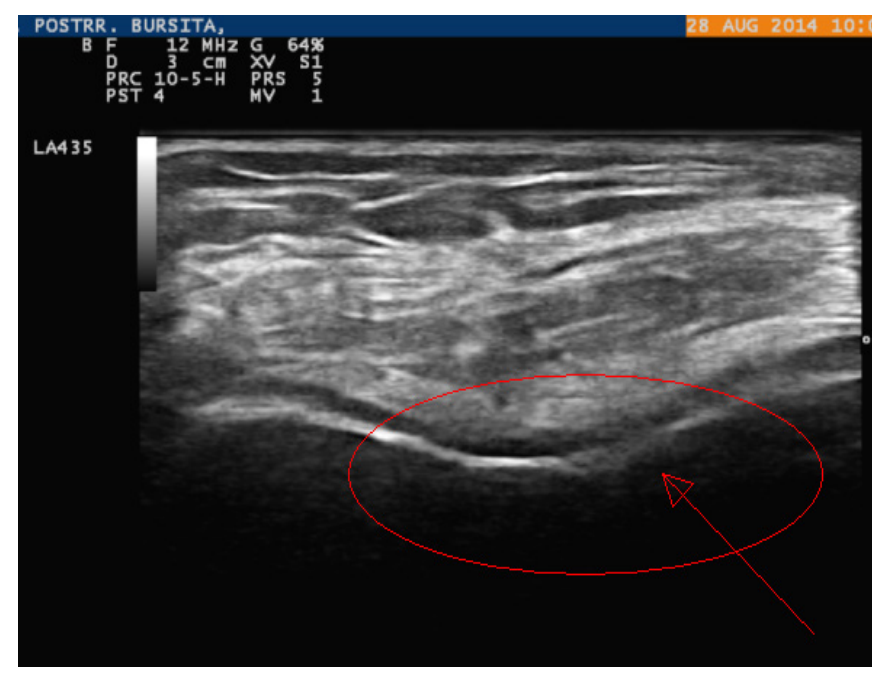

Fig. 2. Hypoechoic hyaline cartilage

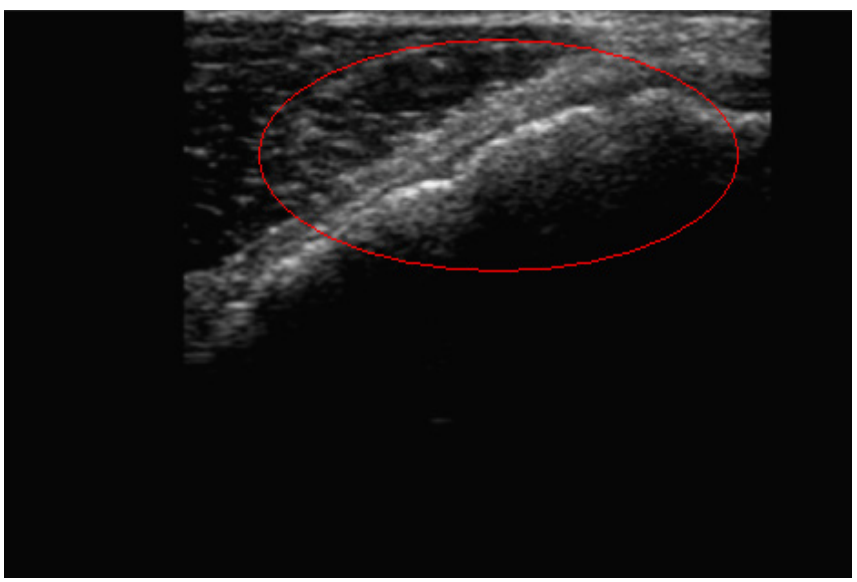

Fig. 4. Involvement medial cartilage

\section{Results}

Twenty-nine patients passed the inclusion criteria. The final ultrasound scores obtained by the ultrasound evaluators weren't significant different $(\mathrm{p}=0.1250$, rs - Spearman: $0.6457 / \mathrm{p}=0.0556$ ), the mean US score being $4.103+/$ 1.263 vs. $3.828+/-1.537$ at the initial evaluation. Same results were obtained at 3 months monitoring (p: 0.2488 , rs - Spearman: $0.7369, \mathrm{p}<0.0001)$ with a mean US score of $5.333+/-0.8660$ vs. $4.778+/-0.8333$. At initial evaluation, a statistically significant difference in the score was noted between the 2 evaluators concerning the joint effusion $(\mathrm{p}=0.0020)$.

No differences were noted in evaluating the joint effusion initially and at the second evaluation ( $p>0.999$, rs/ Spearmann: $0.1890 / \mathrm{p}=0.0833$ ). The same results were obtained in evaluating the presence of hypoecogenity ( $\mathrm{p}$ $>0.9999)$, the thinning of cartilage $(\mathrm{p}=0.5000)$, the osteophytes ( $p>0.3750$, re/Spearman: $-0.05976, p=0.2778$ ), the lateral cartilage (p: 0.3750 , rs/Spearmann: -0.3162 , p $<0.0001)$ or the medial one $(\mathrm{p}=0.2500)$.

Wanting to explore different associations of the lesions followed, we observed a correlation of the medial cartilage involvement with the thinning of it $(\mathrm{p}=0.001)$, the involvement of the lateral cartilage $(\mathrm{p}=0.001)$, the thinning of the cartilage being the most prevalent modification ( $\mathrm{p}$ 
$<0.0001)$. Although, we expected on the medial side the presence of the osteophytes, statistically these changes were mostly seen on the lateral side $(\mathrm{p}<0.0001)$.

Although the ecographic investigation did not show any significant improvements regarding the structural damage of the hyaline cartilage, these findings were not correlated with the functional status of the subjects. By doing a clinical evaluation, using the TegnerLysholm Knee Scoring Scale (Table I), we noticed a slight improvement in the score(day 0; $77+/-6.055$ vs. day 84: $88.71+/-7.410$, $\mathrm{p}=0.00012$ ), especially at 4 weeks intervals, the most significant difference being the pain intensity, limp and swelling of the knee.

We documented mild worsening of knee pain after the injection, in the first 24 hours, in 4 cases, but the pain disappeared spontaneously in the first 2 days. From the twen-

Table I. Tegner Lysholm Knee Scoring Scale

\begin{tabular}{|c|c|c|}
\hline Criteria & Points & Patient Score \\
\hline \multicolumn{3}{|l|}{ Limp (5 points) } \\
\hline None & 5 & $=5$ \\
\hline Slight or periodical & 3 & \\
\hline Severe and constant & 0 & \\
\hline \multicolumn{3}{|l|}{ Support (5 points) } \\
\hline None & 5 & $=5$ \\
\hline Stick or crutch & 2 & \\
\hline Weight-bearing impossible & 0 & \\
\hline \multicolumn{3}{|l|}{ Locking (15 points) } \\
\hline No locking / no catching sensation & 15 & $=15$ \\
\hline Catching sensation, no locking & 10 & \\
\hline Locking occasionally & 6 & \\
\hline Locking frequently & 2 & \\
\hline Locked joint on examination & 0 & \\
\hline \multicolumn{3}{|l|}{ Instabillity ( 25 points) } \\
\hline Never giving away & 25 & $=25$ \\
\hline Rarely during athletic or other severe exercise & 20 & \\
\hline Frequently during athletic or other severe exercise & 15 & \\
\hline Occasionally in daily activities & 10 & \\
\hline Often in daily activities & 5 & \\
\hline Every step & 0 & \\
\hline \multicolumn{3}{|l|}{ Pain (25 points) } \\
\hline None & 25 & $=25$ \\
\hline Inconstant and slight during severe exertion & 20 & \\
\hline Marked during severe exertion & 15 & \\
\hline Marked on or after walking more than $2 \mathrm{~km}$ & 10 & \\
\hline Marked on or after walking less than $2 \mathrm{~km}$ & 5 & \\
\hline Constant & 0 & \\
\hline \multicolumn{3}{|l|}{ Swelling (10 points) } \\
\hline None & 10 & $=10$ \\
\hline On severe exertion & 6 & \\
\hline On ordinary exertion & 2 & \\
\hline Constant & 0 & \\
\hline \multicolumn{3}{|l|}{ Stair climbing (10 points) } \\
\hline No problems & 10 & $=10$ \\
\hline Mild: 0-5 degrees slightly impaired & 6 & \\
\hline One step at a time & 2 & \\
\hline Impossible & 0 & \\
\hline \multicolumn{3}{|l|}{ Squatting (5 points) } \\
\hline No problems & 5 & $=5$ \\
\hline Slightly impaired & 4 & \\
\hline Not beyond 90 degrees & 2 & \\
\hline \multirow[t]{2}{*}{ Impossible } & 0 & \\
\hline & & Total $=100$ \\
\hline
\end{tabular}

ty-nine patients investigated so far, all of them expressed a favorable outcome.

\section{Discussion}

The past several years have been marked by a rapid increase of interest in the use of PRP for the treatment of a wide range of musculoskeletal pathology. Our study revealed that intra-articular injections of PRP mainly showed a reduction of pain and recover function of the kneejoint in patients affected by mild to severe chondropathies, yet these reliefs in patients symptomatology are not correlated with structural changes in the immediate time window. We were unable to find any improvements or worsening concerning the ultrasound alterations during these 3 months investigations. PRP was prepared from autologous blood, so any concerns of allergic reactions were eliminated. We also noticed better clinical scores among the investigated patients, compared to other clinical trials performed in our clinic which used Hyaluronic Acid as an adjuvant therapy in knee OA healing. Further study of PRP in combination with visco-supplementation should also be performed in the future to determine whether ther is any synergistic effect. These preliminary results showed that treatment with PRP injections is safe and has the potential to reduce pain therefore improving life quality among patients with lowdegree articular degeneration. PRP effecton patients with grade III and above chondropathy is questionable.

US gained a lot in the field of diagnosis and monitoring of different diseases, especially the osteoarthritis. The limitation of this method is the missing of a validating scoring system, the means of quantifying the cartilage thinning and last but not the least is the fact that is an operator - dependant technique. So, one of our objectives is to validate a qualitive, easy to reproduce score in knee osteoarthtitis. The imagistic findings showed that US is a reproductable method (no big differences between evaluators), even though at the first evaluation a difference was observed concerning the joint effusion. This might be explained by the fact that the evaluation was a qualitative one, and not quantitative measurements were performed. We consider a noticed effusion if measures more than $4 \mathrm{~mm}$. The senior physician re - measured quantitative the effusions, and reconsidered the evaluation of this specific lesion $[14,15,19]$.

Even though the ultrasound investigations of the knee showed no structural changes in the hyaline cartilage, the aim of the study is to evaluate the non-development of new lesions. No worsening of the cartilage defect and improved clinical scores at 6 months interval may be considered a success and can indicate that PRP therapy is a viable solution for the treatment of initial stages of knee OA. Until now, we didn't observe an evolution of the US score.

Our study is one of the first in our clinic, which is focused on the use of autologous therapy in patients with knee chondropathy, but at this point it has some limitations including the lack of placebo control and short follow-up period. 


\section{Conclusions}

1. In the short term, the local treatment showed no improvement on structural damage, but it improved the overall status of the patients (less pain, improved mobility, a better quality of life) suffering from mild-degree cartilage damage.

2. The application of antilogous PRP is a cost-efficient and safe method in alleviating pain and such improving the quality of life in patients with knee OA.

3. Further studies are needed to confirm these results and to investigate the persistence of the beneficial clinical effects observed.

\section{Acknowlegement}

This paper was published under the frame of European Social Found, Human Resources Development Operational Programme 2007-2013, project no. POSDRU/159/1.5/S/133377

\section{Conflicts of interest}

The authors report no conflicts of interest.

\section{Reference}

1. Curl WW, Krome J, Gordon ES, et al. Cartilage injuries: a review of 31,516 knee arthroscopies. Arthroscopy. 1997;13:456-460.

2. Widuchowski W, Widuchowski J, Trzaska T. Articular cartilage defects: study of 25,124 knee arthroscopies.Knee. 2007;14:177-182.

3. Han B, Woodell-May J, Ponticiello M, et al. The effect of thrombin activation of platelet-rich plasma on demineralized bone matrix osteoinductivity. J Bone Joint Surg Am. 2009;91:1459Y70

4. Landesberg R, Roy M, Glickman RS.Quantification of growth factor levels using a simplified method of platelet-rich plasma gel preparation. J Oral
MaxillofacSurg. 2000;58:297

5. Sanchez M, Anitua E, Azora J, et al. Intra-articular injection of an autologous preparation rich in growth factors for the treatment of knee OA: retrospective kohort study. ClinExpRheumatol. 2008;26:910Y3.

6. Kellett CF, Boscainos PJ, Gross AE. Surgical options for articular defects of the knee. Expert Rev Med Devices. 2006;3:585-593.

7. Buckwalter JA, Brown TD. Joint injury, repair, and remodeling: roles in post-traumatic osteoarthritis. ClinOrthopRelat Res. 2004;423:7-16.

8. Ochi M, Uchio Y, Kawasaki K, et al. Transplantation of cartilage-like tissue made by tissue engineering in the treatment of cartilage defects of the knee. J Bone Joint Surg Br. 2002;84:571-578.

9. Mankin HJ. The response of articular cartilage to mechanical injury.JBone Joint Surg Am. 1982;64:460-466.

10. Anitua E, Andia, I, Ardanza B, et al. Autologous platelets as a source of proteins for healing and tissue regeneration. ThrombHaemost. 2004;91:4Y15

11. Giannini S, Vannini F, Timoncini A, et al. The treatment of severe chondropaties of the knee with autologous platelet rich plasma injections: Preliminary results. Osteoarthr Cartilage. 2008;16:126Y7

12. Spakova' T, Rosocha J, Lacko M, Harvanova' D, Gharaibeh A. Treatment of knee joint osteoarthritis with autologous platelet-rich plasma in comparison with hyaluronic acid. Am J Phys Med Rehabil. 2012;91:411Y417.

13. Mishra A, Pavelko T: Treatment of chronic elbow tendinosis with buffered platelet-rich plasma. Am J Sports Med. 2006;34:1774Y8

14. Möller I, Bong D, Naredo E, et al. Ultrasound in the study and monitoring of osteoarthritis, Osteoarthritis and Cartilage. 2008;16:S4-S7

15. lagnocco A. Imaging the joint in osteoarthritis: a place for ultrasound?, Best Practice and Research Clinical Rheumatology. 2010;24:27-38

16. Keen HI, Wakefield RJ, Conaghan PG. A systematic review of ultrasonograpy in osteoarthritis, ARD. 2009;68:611-617

17. Backhaus M, Brumester G.H., Gerber T, et al. Guidelines for musculoskeletal ultrasound in rheumatology, ARD. 2001;60:641-649

18. Wakefield RJ, Balint PV, Szkudlarek M, et al. Musculoskeletal ultrasound including definitions of ultrasonographic pathology, Journal of Theumatology. 2005;32:485-2487

19. Abraham AM, Goff I, Pearce MS, Francis RM, Birell F. Reliability and validity of ultrasound imaging of features of knee osteoarthritis in the community, BMC Musculoskeletal Disorders. 2011;2:70 